\title{
UVCS/SOHO observations of a CME-driven shock: Consequences on ion heating mechanisms behind a coronal shock
}

\author{
S. Mancuso ${ }^{1,2}$, J. C. Raymond ${ }^{1}$, J. Kohl ${ }^{1}$, Y.-K. Ko ${ }^{1}$, M. Uzzo ${ }^{1}$, and R. Wu ${ }^{1}$ \\ 1 Harvard-Smithsonian Center for Astrophysics, 60 Garden Street, MS 50, Cambridge, MA 02138, USA \\ 2 Present address: Osservatorio Astronomico di Torino, Strada Osservatorio 20, Pino Torinese 10025, Italy
}

Received 3 August 2001 / Accepted 15 November 2001

\begin{abstract}
We report the observation of a $1100 \mathrm{~km} \mathrm{~s}^{-1}$ CME-driven shock with the UltraViolet Coronagraph Spectrometer (UVCS) telescope operating on board SOHO on March 3, 2000. The shock speed was derived from the type II radio burst drift rate and from UVCS observations that can yield the density profile just before the passage of the shock. A CME projected speed of $920 \mathrm{~km} \mathrm{~s}^{-1}$ was deduced from the Large Angle Spectrometric Coronagraph (LASCO) white light images, indicating that the CME leading edge was lagging behind at about $20 \%$ of the shock speed. The spectral profiles of both the O VI and Ly $\alpha$ lines were Doppler dimmed and broadened at the passage of the shock by the emission from shocked material along the line of sight. The observed line broadening for both protons and oxygen ions was modeled by adopting a mechanism in which the heating is due to the nondeflection of the ions at the shock ramp in a quasi-perpendicular shock wave. This specific ion heating model was able to reproduce the observed spectroscopic properties of the shocked plasma.
\end{abstract}

Key words. Sun: corona, UV radiation, radio radiation - shock waves - magnetohydrodynamics (MHD)

\section{Introduction}

Coronal type II solar radio bursts represent an important diagnostic for the passage of magnetohydrodynamic (MHD) shocks through the solar corona. The type II radio emission is generated by the shock-excited plasma emission at the electron plasma frequency $f_{\mathrm{pe}}=$ $0.00898 \sqrt{n_{\mathrm{e}}\left(\mathrm{cm}^{-3}\right)} \mathrm{MHz}$ and its harmonic (Wild 1950; Nelson \& Melrose 1985). The frequency drift rate can be combined with a model of coronal density versus height for an estimate of the shock speed.

It is usually assumed that coronal shocks originate in proximity of a flare as a result of a blast wave or that they are piston-driven ahead of the leading edge of a coronal mass ejection (CME) with sufficiently high initial speed at low heights. However, the determination of their origin is not straightforward since the speeds, angular extents, and locations of the shocks relative to the CMEs are poorly known due to the fact that radio spectrograph observations have no spatial resolution. Although radioheliographs can provide information on the shock location, observations of coronal radio bursts with such instruments are scarce and affected by strong refraction at the lower heights. Moreover, the type II radio emission yields no

Send offprint requests to: S. Mancuso,

e-mail: mancuso@to.astro.it information on the mechanism of heating of the shocked coronal plasma.

The UltraViolet Coronagraph Spectrometer (UVCS) telescope aboard the Solar and Heliospheric Observatory (SOHO) spacecraft is able to observe the spectroscopic signature of the passage of a coronal shock. UVCS observations have not only the potential of detecting the spatial location and timing of the shock (compared to that of the CME leading edge) but can also yield unique information on the heating process of the shocked plasma. Finally, UVCS observations provide a unique opportunity to determine the background pre-shocked coronal density profile in which the shock propagates.

The possibility of spectroscopically detecting a CMEdriven coronal shock with the SOHO spacecraft has been already discussed by Kahler et al. (1999). Recently, Raymond et al. (2000) presented the first evidence of a solar coronal shock observed with an ultraviolet solar spectrograph on June 11, 1998.

In this paper we present refined ultraviolet spectroscopy of a coronal shock observed by the UVCS telescope on March 3, 2000 and we apply a specific model of ion heating to interpret the observed oxygen line broadening after the shock passage through the slit of the spectrometer. We describe the observations in Sect. 2. In Sect. 3 we derive the main physical parameters of the 


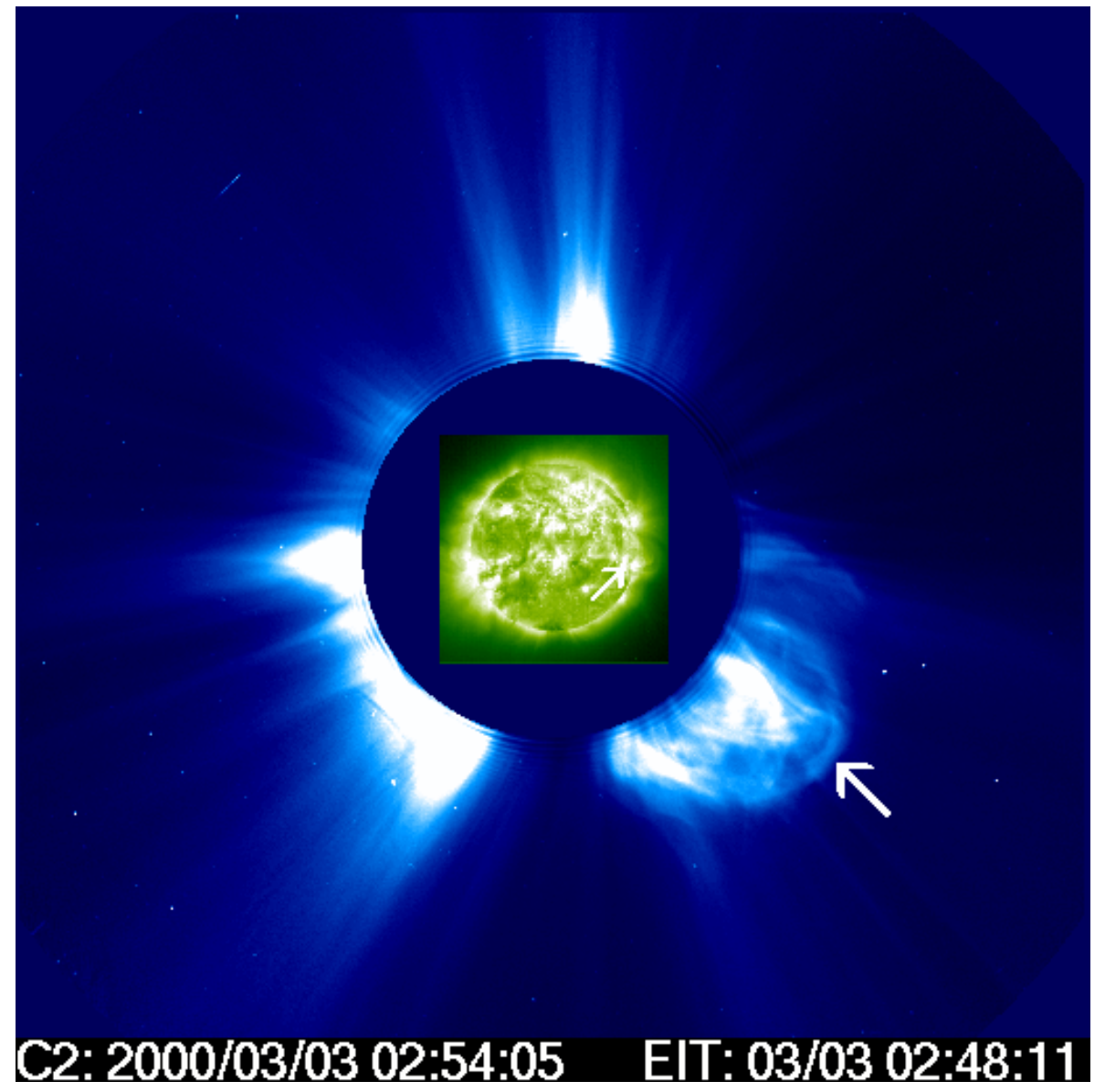

Fig. 1. Composite image using the EIT $195 \AA$ image taken at 2:48 UT, and the LASCO C2 white-light image taken at 2:54 UT on March 3, 2000. The arrows indicate respectively the position of the active region NOAA 8882 on the disk and the position of the leading edge of the CME front.

shock. In Sect. 4 we model the observed line broadening by applying a specific mechanism of ion heating for the shocked plasma. Finally, we summarize our results in Sect. 5 .

\section{Observations}

The event on 2000 March 3 was observed by UVCS, LASCO C2 and C3, and EIT operating on board SOHO. The UVCS (Kohl et al. 1995) instrument is designed to observe the extended solar corona from 1.4 to about $10 R_{\odot}$. Coronal spectra are acquired in two spectrometer channels: the LY $\alpha$ channel, which covers the range of 1160 to $1350 \AA$ and the OVI channel, covering the range of 940$1123 \AA$. Our observations utilized the O VI channel that is optimized for the study of the O VI doublet 1031.91 and $1037.61 \AA$. A convex mirror between the grating and the detector in the $\mathrm{O}$ VI channel images spectral lines in the range 1160-1270 $\AA$ onto the O VI detector, allowing the detection of the redundant Ly $\alpha$ at $1215.67 \AA$. Table 1 lists the spectral lines observed by UVCS which were used in this work.

UVCS observed the shock during the daily synoptic program beginning with a scan at the west limb at radii $1.5,1.6,1.7,1.8,2.0,2.3,2.8$, and $3.5 R_{\odot}$. The observation times at these heights were 400, 400, 540, 540, 720, 900,1520 , and $2080 \mathrm{~s}$ respectively, broken into 180 or $200 \mathrm{~s}$ exposures from 2.04 to 4:19 UT. The slit width was
Table 1. Spectral lines observed.

\begin{tabular}{ccc}
\hline \hline$\lambda_{\text {ID }}(\AA)$ & Identification & $\log T_{\mathrm{m}}$ \\
\hline 499.37 & Si XII $2 \mathrm{~s}^{2} \mathrm{~S}_{1 / 2}-2 \mathrm{~s} 2 \mathrm{p}^{2} \mathrm{P}_{3 / 2}$ & 6.3 \\
1031.91 & O VI $2 \mathrm{~s}{ }^{2} \mathrm{~S}_{1 / 2}-2 \mathrm{~s} 2 \mathrm{p}{ }^{2} \mathrm{P}_{3 / 2}$ & 5.5 \\
1037.61 & O VI $2 \mathrm{~s}{ }^{2} \mathrm{~S}_{1 / 2}-2 \mathrm{~s} 2 \mathrm{p}^{2} \mathrm{P}_{1 / 2}$ & 5.5 \\
1215.67 & Ly $\alpha$ & 4.1 \\
\hline
\end{tabular}

100 microns $\left(28^{\prime \prime}\right)$ with spatial binning along the slit of $21 \operatorname{arcsec}(3$ pixels). Spectral binning was 3 pixels for the primary and 2 pixels for the redundant channel (yielding a spectral resolution of 0.2979 and $0.1830 \AA$ respectively). In the data reduction we followed the standard techniques described in Kohl et al. (1997, 1999). We used the UVCS Data Analysis Software (DAS) for wavelength and intensity calibration, and removal of image distortion. The uncertainties in the O VI line intensities are due to photon counting statistics, background subtraction, and radiometric calibration (estimated to be 12\%-15\%) (Gardner et al. 1996). The shock entered the slit at the first exposure of the three centered at $1.7 R_{\odot}$, taken between $2: 19$ and 2:22 UT. In the rest of the paper we will refer to these three exposures as exposures 1,2 and 3 .

A CME with a bright front in the southwest sector of the Sun was observed in white light on the same day (Fig. 1) with the Large Angle Spectrometric Coronagraph (LASCO) (Brueckner et al. 1995). The instrument consists 


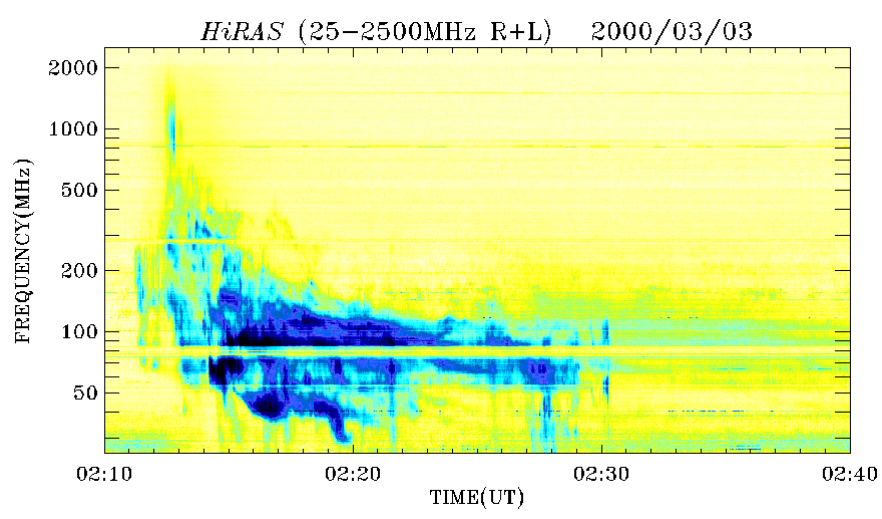

Fig. 2. Dynamic spectrum from the Hiraiso radio spectrograph showing metric type II emission. The radio intensity is grey scale coded where dark means bright emission.

of three optical coronagraphs $(\mathrm{C} 1, \mathrm{C} 2$, and $\mathrm{C} 3)$ with overlapping fields of view of $1.1-3.0,2.0-6.0$, and $3.7-32 R_{\odot}$ respectively.

This event was also associated with an $\mathrm{H} \alpha$ solar flare with an onset at 02:11 UT and a maximum at 02:13 UT (Solar Geophysical Data) that was located in the active region NOAA 8882 at S15W60. An X-ray event of class M3.8 from the same active region was observed by the GOES instrument from 02:08 to 02:22 UT, with peak at 02:14 UT. The flare was visible in the Fe XII line (195 A, corresponding to a temperature of about $1.5 \mathrm{MK}$ ) with the Extreme Ultraviolet Imaging Telescope (EIT) (Delaboudinière et al. 1995). This instrument is a normalincidence telescope that images the solar disk with a field of view extending to the inner corona in four selected wavelength regions corresponding to strong EUV emission lines. Around the same time a second $\mathrm{H} \alpha$ flare was reported from active region NOAA 8886 located at S14W76 with peak at 2:12 UT.

The metric radio emission in the frequency range from 25 to $2000 \mathrm{MHz}$ associated with this event is shown in Fig. 2. This radio dynamic spectrum, from the Hiraiso Radio Spectrometer (Kondo et al. 1995), covers the time period from 02:10 to 02:40 UT. The same radio event was observed in Australia by the Culgoora Radiospectrograph (Prestage et al. 1994) and the Bruny Island Radio Spectrometer (BIRS) (Erickson 1997). The dynamic spectrum for this event shows rather complex metric type II radio emissions, exhibiting an intense backbone at the fundamental and harmonic emission, multiple lanes, and herringbone structure. The backbone and herringbones are respectively interpreted as the radio signature of a shock wave that travels through the solar corona at super-Alfvénic velocity and electron beams accelerated at the shock wave. The fast-frequency-drifting radio emissions beginning at 2:12 UT are from type III radio bursts associated with the flare onset. The observed type II burst is most probably a superposition of emission coming from the shock in a quite inhomogeneous corona: the shock propagates outwards encountering different densities at different sections of the shock resulting in multiple emission lanes (McLean 1967).

\section{Data analysis}

The main goal of this paper is to obtain an interpretation of the observed broad profiles in the light of known mechanisms of ion heating. In order to achieve this goal, we will need an estimate of some shock parameters, such as the shock speed and the compression ratio. Furthermore, we will need an estimate of the CME speed to investigate the nature of the shock origin (blast wave driven or pistondriven) and an estimate of the electron density profile to derive the shock speed from the observed frequency drift rate of the metric type II radio emission.

\subsection{CME speed}

The CME speed was estimated from white light data by comparing the position of the leading edge of the coronal mass ejection as seen by LASCO C2 and C3 at different times. The error on these positions is typically around 10 pixels in $\mathrm{C} 2$ which corresponds to about $0.1 R_{\odot}$. The coronagraph gives us a two-dimensional view of the corona where features are projected onto the plane of the sky, so we may have underestimated the true speed. However, the active region group associated with the CME was near the limb and consequently projection effects should not be important. This is also confirmed by an analysis of the Doppler shift of the broad components of the O VI doublet after the passage of the shock, from which it is evident that the bulk velocity along the line of sight must have been well below $100 \mathrm{~km} \mathrm{~s}^{-1}$. From a straight line fit to the height-time data (Fig. 3), we estimate a projected speed of $920 \mathrm{~km} \mathrm{~s}^{-1}$ above about $3 R_{\odot}$. The height-time data are well fit by the straight line, suggesting no significant acceleration above this height. The projected lift-off time of the CME, obtained by simply extrapolating the best straight-line fit back to the surface of the Sun, is consistent with the onset time of the X-ray event. However, we have no specific reason to assume a constant speed under the coronagraph occulting disk, and we should anticipate some significant acceleration near the Sun that would put the onset of the CME somewhat earlier than the onset time extrapolated from the straight line fit in Fig. 3.

\subsection{Density estimate}

The shock dynamics implied by the metric type II radio emission can be determined by knowing the drift rate at the frequency of observation and the electron density gradient in the corona at the plasma level corresponding to the observing frequency. The first two quantities are directly determined, but the electron density is usually taken from a model. Consequently, the derivation of the shock speed is strongly dependent on the assumed density model. UVCS observations of the pre-shocked plasma 


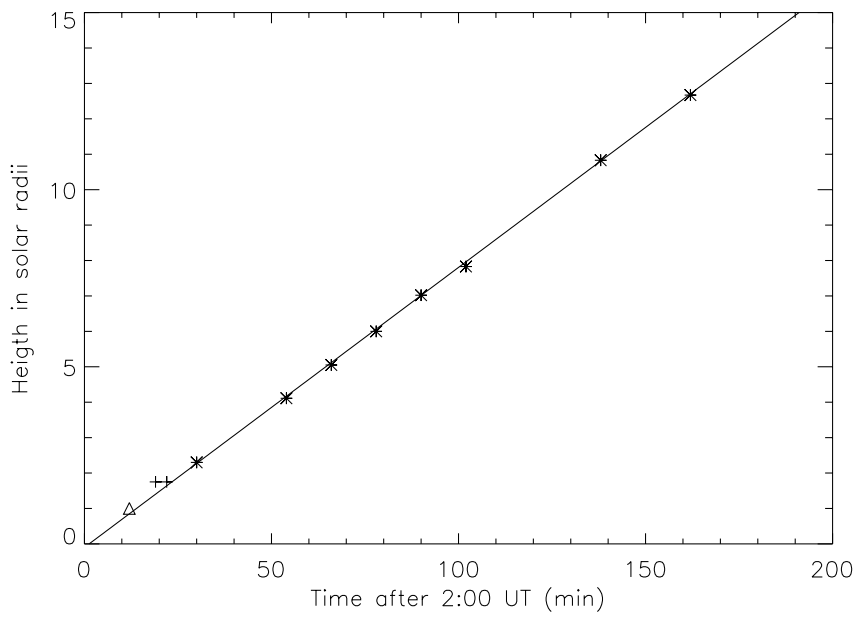

Fig. 3. Height-time data for the LASCO CME observed on March 3, 2000. The straight line is the linear fit obtained assuming a constant projected velocity of $920 \mathrm{~km} \mathrm{~s}^{-1}$. The triangle locates the time of onset of the flare. The stars represent estimates of the leading front of the CME projected in the plane of the sky (from LASCO observations). The crosses represent the interval time for exposure 1 where the shock was first detected.

can be used to obtain a reliable estimate of the density gradient just before the passage of the shock.

Ultraviolet lines in the lower corona form essentially by collisional excitation followed by radiative de-excitation. The intensity of collisionally excited lines depend on electron densities $n_{\mathrm{e}}$ via the line-of-sight integral of $n_{\mathrm{e}}^{2}$. At higher levels, and for bright enough exciting chromospheric radiation, lines form also via resonant scattering of radiation: this is the dominant process for the hydrogen Ly $\alpha$, while both components need to be considered for the O VI lines. The Ly $\alpha$ line is produced by resonance scattering (Gabriel et al. 1971) and is therefore subject to Doppler dimming (Hyder \& Lites 1970). The two lines of the O VI resonance doublet are excited in part by collisions with free electrons, in part by transition region radiation (Kohl \& Withbroe 1982), therefore their radiative components can be affected by Doppler dimming. The radiative contribution depends on the width of the coronal absorption profile, which is set by the velocity distribution of the absorbing ions in the direction of the incoming radiation. The O VI ions, because of their larger mass and thus much narrower absorption profile, are much more sensitive to this effect than the H I atoms. However, for a nearly static plasma, as we expect above the active region and below $2 R_{\odot}$, Doppler dimming is negligible and the coronal electron density can be directly estimated from the observed ratio of the radiative and collisional components of the O VI $1032 \AA$ line (Noci et al. 1987). The O VI intensity ratio is given by:

$$
\frac{I_{\mathrm{rad}}(1032)}{I_{\mathrm{col}}(1032)}=\frac{5.75 \times 10^{2} \lambda^{2} \exp \left(E_{1 k} / k_{\mathrm{B}} T_{\mathrm{e}}\right) \sqrt{T_{\mathrm{e}}} I_{\mathrm{disk}} \Delta \Omega}{\pi g N_{\mathrm{e}} \sqrt{\Delta \lambda_{\text {cor }}^{2}+\Delta \lambda_{\mathrm{ex}}^{2}}}(1)
$$

where $\lambda=1031.912 \AA, E_{1 k}=h c / \lambda$ is the energy difference between the levels involved in the transition, $g=1.13$ is the Gaunt factor, $\Delta \lambda_{\mathrm{ex}}=0.10 \AA$ is the $1 / e$ half width of the exciting line from the lower atmosphere, $\Delta \lambda_{\text {cor }}=0.11 \AA$ is the $1 / e$ half-width of the coronal absorption profile and $\Delta \Omega=2 \pi\left[1-\sqrt{\left.1-\left(R_{\odot} / r\right)^{2}\right)}\right]$ is the solid angle subtended by the solar disk at a distance $r$. $I_{\text {disk }}$ is the intensity of the line integrated over the disk. Our estimate of $866.2 \mathrm{erg} \mathrm{cm}^{-2} \mathrm{~s}^{-1} \mathrm{sr}^{-1}$ was derived by logaveraging the intensities measured by Vernazza \& Reeves (1978) for a quiet area and for an active region.

In order to determine the electron density from the above formula, we also need an estimate of the electron temperature $T_{\mathrm{e}}$. We assumed that at the low heights of our observations the density is high enough that the kinetic temperatures of the electrons and protons are the same, i.e. $T_{\mathrm{e}}=T_{\mathrm{p}}$. The proton temperature $T_{\mathrm{p}}$ was estimated from the Doppler width of the Ly $\alpha$ line assuming that the neutral hydrogen and the protons are coupled through charge exchange below $2 R_{\odot}$ and that therefore they have the same temperature. The two intensity components have been derived from the simple relationship between the intensities of the collisionally excited and radiatively excited components of the O VI doublet (Noci et al. 1987):

$I_{\text {col }}(1032) / I_{\text {col }}(1037)=2$

$I_{\text {rad }}(1032) / I_{\text {rad }}(1037)=4$

with the total intensity given by $I_{\text {tot }}=I_{\text {rad }}+I_{\text {col }}$.

From the analysis of the observed spectra, we derived the following density law:

$n_{\mathrm{e}}(r)=1.26 \times 10^{8} r^{-4.5}$

in the range between $1.5-1.8 R_{\odot}$. It is interesting to notice that this density law roughly corresponds to 0.85 times a Newkirk (1961) model at $1.8 R_{\odot}$. This latter model is often used in literature (multiplied by a factor ranging between one and a few) for the evaluation of the shock speed in the lower corona.

We point out that a major uncertainty in the above estimate for the electron density profile derives from the lack of simultaneous measurements of the O VI $1032 \AA$ line on the disk, as we do not really know how large a variation we can expect in the O VI $1032 \AA$ line disk intensity along the solar cycle and the exact contribution of the active region group on the intensity of the solar disk. However it is unlikely for the actual O VI flux to be less than $40 \%$ above the quiet Sun value of $305 \mathrm{erg} \mathrm{cm}^{-2} \mathrm{~s}^{-1} \mathrm{sr}^{-1}$ (Vernazza \& Reeves 1978) since the Ly $\alpha$ flux from the whole Sun is only about $40 \%$ times the solar minimum quiet Sun value (Woods et al. 1998). Given that the actual flux is also unlikely to be as high as the active region surface flux, an uncertainty of $\pm 40 \%$ for the value of the O VI flux would be a reasonable estimate.

\subsection{Shock speed and compression ratio}

The radial velocity of the shock can be deduced from the drift rate of the associated radio signature of the drifting lanes in the spectrograph. The instantaneous drift 
rate at the time corresponding to the passage of the shock front through the slit, positioned at $1.7 R_{\odot}$, was $D_{\mathrm{f}}=-0.065 \mathrm{MHz} \mathrm{s}^{-1}$ for the fastest drifting lane. The relationship between the drift rate $D_{\mathrm{f}}$ measured at the frequency $f_{\text {pe }}$ and the radial velocity of the shock $V_{\mathrm{s}}$ is given by

$V_{\mathrm{s}}=\frac{2 D_{\mathrm{f}}}{f_{\mathrm{pe}}} \frac{n_{\mathrm{e}}}{\mathrm{d} n_{\mathrm{e}} / \mathrm{d} r}$.

The estimated shock speed at the height of the slit is $V_{\mathrm{s}}=$ $1100 \mathrm{~km} \mathrm{~s}^{-1}$. This estimate is in good agreement with the theory of piston-driven shocks, according to which the shock speed is about $20 \%$ higher than the piston velocity $\left(920 \mathrm{~km} \mathrm{~s}^{-1}\right)$ and travels about $20 \%$ ahead of the piston (Maxwell et al. 1985). We need to mention that projection effects could actually change this result, in the sense that the CME speed derived from coronograph data could be underestimated. However, if the CME would have travelled much away respect to the plane of the sky, we would have noticed considerable Doppler shifts in the following exposures higher in the corona.

The compression ratio was estimated from the observed splitting of the fundamental and harmonic bands (Smerd et al. 1975). The type II radio burst shows in fact episodes of band-splitting that, although not clearly evident from Fig. 2, can be detected after a careful inspection of the radio spectra. According to the model of Smerd et al. (1975), we identified the frequency of the lowerfrequency component of a split band with the plasma frequency just ahead of the shock front, while the frequency of the upper-frequency component is identified with the plasma frequency just behind the shock. The observed frequency band splitting at the time of the passage of the shock through the slit implies a density jump of about 1.8 .

\section{Interpretation and modeling}

\subsection{Interpretation of the observed UV spectra}

The southern end of the UVCS slit (positioned at $1.7 R_{\odot}$, tangent to the west limb) detected the passage of part of the northern flank of the shock front in exposure 1 and following exposures. In Fig. 4 we sketch a possible shock geometry together with the slit position at the time of the passage of the shock through the slit (Fig. 4a) and in the following two exposures (Fig. 4b). For simplicity we assumed a hemispherical shock centered on the surface of the Sun propagating at the speed derived in Sect. 3.3.

Due to the small portion of shocked gas detected in the first exposure along the line of sight, most of the emission in this exposure is attributable to foreground and background material along the line of sight and unshocked material along the shock normal. As a working hypothesis we will then assume that the emission from the shocked gas in exposure 1 is negligible. This assumption is necessary in order to simplify the following analysis, since we do not have exposures taken at the same height before the passage of the shock front.
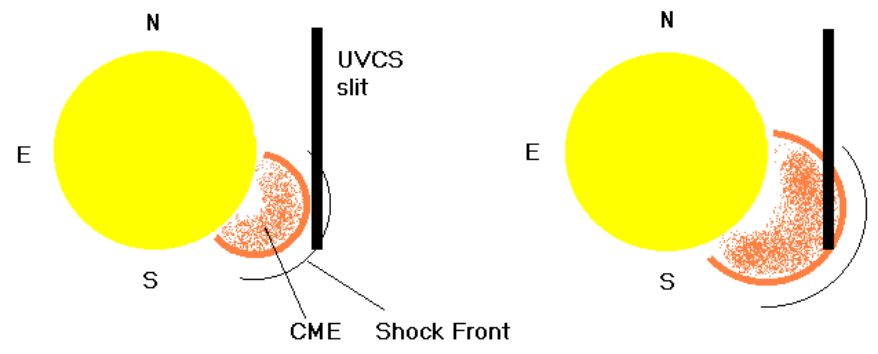

a)

b)

Fig. 4. Sketch of the possible geometry of the passage of the shock through the slit on March 3, 2000: a) at exposure 1 and $\mathbf{b}$ ) at exposures 2 and 3 . We assumed an hemispherical expansion of the shock in front of the CME leading edge.

The two major features observed in the UV spectra after the passage of the shock are the dimming of the line intensities and the presence of broad wings in the oxygen profiles.

The Ly $\alpha$ and O VI $1032 \AA$ line intensities at the southernmost portion of the slit at $1.7 R_{\odot}$ (corresponding to an height of about $1.8 R_{\odot}$ ) drop of about a factor of 1.3 between exposures 1 and 2 (see Table 2). This is mainly a consequence of the Doppler dimming effect, since the outflow velocity of the coronal plasma increases at the passage of the shock, so that fewer particles are able to scatter the disk radiation. As for the O VI $1032 \AA$ line, the radiative component is practically washed out at the high speed of the shock. Moreover, the higher electron temperature in the shocked plasma ionizes more of the neutral hydrogen while the O VI ions are excited to higher ionization states, which reduces the line intensities in both cases.

The most interesting feature that is observed spectroscopically after the shock passage is an overall increase of the width of the spectral lines and the clear presence of broad wings in the oxygen line profiles. A probable cause for the observed line broadening is the heating of the shocked plasma (Raymond et al. 2000), but other possible causes may be non-thermal effects from ions energized at the shock front (Kahler et al. 1999) and the expansion of the CME front. The line broadening of the oxygen line profiles is expected from in situ measurements that show a larger heating of the less abundant ion species in the solar wind respect to the protons downstream of an interplanetary shock (e.g., Berdichevsky et al. 1997). The downstream ion temperature can be several tens of times higher than that in the upstream region.

The neutral atoms should remain unaffected by the passage of the MHD collisionless shock. In fact, the neutral component of the plasma can only interact with the hot plasma through collisions and, because of the long thermalization time, the coupling between protons and electrons is only effective after several tens of minutes. However, the process of resonant charge transfer between fast shocked protons and slow hydrogen atoms, that has a typical timescale of $1 \mathrm{~s}$ in the shocked plasma, can be 
effective in producing a fast thermalization of the neutrals resulting in a Maxwellian distribution of neutrals displaying the thermal properties of the post-shock ions. These neutrals can then emit Ly $\alpha$ in the form of a broad peak centered upon the post-shock ionic velocities. As a consequence, the observed broad Ly $\alpha$ profile can be interpreted as a direct measure of the proton temperature behind the shock. By assuming that the protons in the shock are only heated by adiabatic compression $\left(T_{2} / T_{1}=\left(n_{2} / n_{1}\right)^{2 / 3}\right)$, we should observe an increment of the temperatures of a factor 1.6. The width of the observed $\operatorname{Ly} \alpha$ profile, corresponding to a post-shock temperature of $T \approx 2.5 \times 10^{6} \mathrm{~K}$, is in fact apparently consistent with a thermal broadening mechanism due to adiabatic compression of the proton gas and charge exchange with the neutrals. However, the oxygen temperature increase across the shock cannot be explained in terms of adiabatic compression, since the width of the post-shock spectral lines would be much thinner than observed. It is necessary to model the oxygen line broadening with a different mechanism.

\subsection{Application of an ion heating model}

Our observations are obtained at low coronal heights with the transient originating in a closed active region magnetic structure. The structure of the magnetic field region above such a region should remain quasi-perpendicular to the shock normal. In a mechanism proposed by Lee et al. (1986), the heating at a quasi-perpendicular shock is due to the nondeflection of ions at the shock ramp. The nonadiabatic motion of the directly transmitted ions in a quasi-perpendicular shock wave can in fact result in a large increase of the ion kinetic temperature transverse to the ambient magnetic field in the downstream plasma. As a result of the nonadiabatic motion across the thin shock ramp, part of the upstream normal flow velocity is converted to the gyration velocity in the downstream region, which provides an effective ion heating. The ions, after crossing the shock ramp, gyrate and contribute to the downstream heating so that the gyration velocity $v_{\mathrm{g}}$ can be considered as the ion perpendicular thermal velocity $v_{\text {th }}$. According to this mechanism, the downstream gyration speed depends on the mass/charge ratio $M / Q$ as (Lee \& Wu 2000):

$\frac{v_{\mathrm{g}}}{V_{\mathrm{s}}}=\left|\sqrt{1-\alpha \frac{Q}{M}}-\frac{1}{X}\right|$

where $X$ is the observed compression ratio, $\alpha=$ $e \Delta \phi /\left(m_{\mathrm{p}} V_{\mathrm{s}}^{2} / 2\right) \approx 0.45$ (Lee et al. 1987), and $\Delta \phi$ is the electric potential jump at the shock ramp.

By inserting in expression (6) the shock parameters estimated in Sect. 3.3, we obtain $v_{\mathrm{g}} \sim v_{1 / \mathrm{e}}=400 \mathrm{~km} \mathrm{~s}^{-1}$ $\left(T_{\text {kin }}=1.6 \times 10^{8} \mathrm{~K}\right)$ for the oxygen ions and $v_{\mathrm{g}} \sim v_{1 / \mathrm{e}}=$ $200 \mathrm{~km} \mathrm{~s}^{-1}\left(T_{\text {kin }}=2.45 \times 10^{6} \mathrm{~K}\right)$ for the protons. The amount of proton heating is therefore consistent with the observed broadening of the Ly $\alpha$ profile. More importantly,

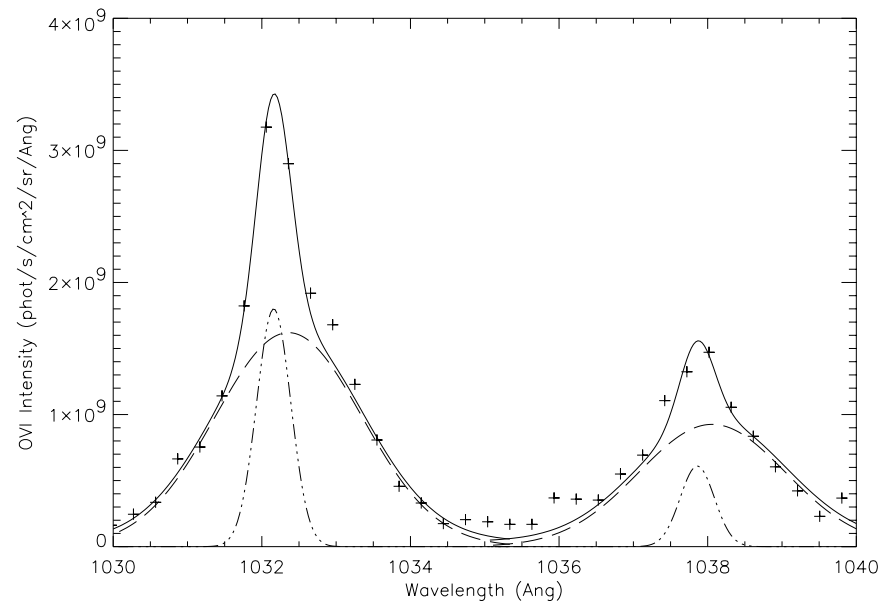

Fig. 5. UVCS 2000 March 3 observations of O VI $\lambda 1032$ and $\lambda 1037$ profiles at $1.7 R_{\odot}$ in the time interval $02: 22: 43-$ 02:25:43 UT (exposure 2). The intensity has been averaged over $9^{\prime}$ between $360^{\prime \prime}$ and $906^{\prime \prime}$ in the southern portion of the slit. Curve models for two Gaussians plus a constant background are shown for each of the two lines. The data points are shown by crosses, and the model profile by a solid line. The dashed curves represent a possible signature of the passage of the shocked gas through the slit. The dash-dotted curves represent background and foreground non-shocked material.

the amount of heating for the oxygen ions is also consistent with the observed broadening of the O VI resonance lines.

In order to evaluate the goodness of such a model, we modeled each of the two oxygen lines in exposure 2 by two Gaussians plus a constant background. Figure 5 shows the observed O VI doublet profile averaged over a $9^{\prime}$ spatial bin along the southern portion of the slit, corresponding to a distance of about $1.8 R_{\odot}$ from the center of the Sun. The observed line profiles show broad wings, which allows two components in the profile to be distinguished: a broad component (dashed line) corresponding to the shocked plasma, and a narrow component (dashdotted line) corresponding to foreground and background unshocked plasma. We kept the O VI $\lambda 1032 / \lambda 1037$ intensity ratio equal to 3.2 (as in exposure 1) for the narrow component since it represents static material along the line of sight with a strong radiative component. On the other hand, the intensity ratio for the broader Gaussian was assumed to be 1.75 due to the fact that the radiative component of the oxygen lines is washed out at the high post-shock velocities of the plasma, while the pumping of the O VI $1037 \AA$ line from the C II $1037.0 \AA$ and $1036.4 \AA$ lines keeps this ratio lower than 2 (Li et al. 1998; Dodero et al. 1998). In Table 2 we summarize the parameters used for the above model.

Because of the low statistics for the Si XII $499.4 \AA$ line, we could not model the observed profiles for this line with a sum of two Gaussians. The Si XII intensity was enhanced by a factor about 2 respect to the intensity (expressed in counts) in exposure 1 (see Table 3). One reason for this enhancement is the higher post-shock electron temperature that increases the number of ions in this ionization 
Table 2. O VI line intensities.

\begin{tabular}{cccccccc}
\hline \hline Exposure & Component & $\begin{array}{c}I_{1032} \\
\left(10^{8} \mathrm{ph} \mathrm{cm}^{-2} \mathrm{~s}^{-1} \mathrm{sr}^{-1}\right)\end{array}$ & $\begin{array}{c}I_{1037} \\
\left(10^{8} \mathrm{ph} \mathrm{cm}^{-2} \mathrm{~s}^{-1} \mathrm{sr}^{-1}\right)\end{array}$ & $\begin{array}{c}I_{1032} / I_{1037} \\
\lambda_{1032} \\
(\AA)\end{array}$ & $\begin{array}{c}\lambda_{1037} \\
(\AA)\end{array}$ & $\begin{array}{c}1 / e \text { Width } \\
\left(\mathrm{km} \mathrm{s}^{-1}\right)\end{array}$ \\
\hline \multirow{2}{*}{1} & unshocked & 41.6 & 13.0 & 3.2 & 1032.14 & 1037.84 & 70 \\
& shocked & 21.3 & 12.0 & 1.75 & 1032.36 & 1038.06 & 400 \\
2 & unshocked & 10.0 & 3.1 & 3.2 & 1032.16 & 1037.86 & 70 \\
& shocked & 39.8 & 22.5 & 1.75 & 1032.36 & 1038.06 & 400 \\
3 & unshocked & 11.1 & 3.5 & 3.2 & 1032.14 & 1037.84 & 70 \\
& shocked & 24.4 & 13.8 & 1.75 & 1032.16 & 1037.86 & 400 \\
\hline
\end{tabular}

Table 3. Ly $\alpha$ and Si XII line intensities.

\begin{tabular}{ccc}
\hline \hline Exposure & $\begin{array}{c}\text { Ly } \alpha \\
\left(10^{8} \mathrm{ph} \mathrm{cm}^{-2} \mathrm{~s}^{-1} \mathrm{sr}^{-1}\right)\end{array}$ & $\begin{array}{c}\text { Si XII } \\
(\text { counts })\end{array}$ \\
\hline 1 & 901 & 126 \\
2 & 711 & 234 \\
3 & 672 & 194 \\
\hline
\end{tabular}

stage. Moreover, the intensity of the Si XII $499.4 \AA$ line is dominated by collisional excitation and is not affected by Doppler dimming at the densities estimated from our observations. The doubling of the Si XII intensity suggests a density increase of about $40 \%$, a figure that is compatible with the fact that we also observe the contribution of foreground and background material along the line of sight.

\subsection{Possible contribution of CME material to the observed profiles}

Although the model presented in Sect. 4.2 is very satisfactory in reproducing the observed post-shock line profiles, an alternative interpretation for the broad wings could be the presence of CME material expanding in a thick shell with components of the velocity along the line of sight. This contribution is hardly quantifiable and subject to major uncertainties deriving from the fact that we have no direct information on the shape of the CME along the line of sight and that the leading edge position height may have been somewhat underestimated as extracted from the LASCO images.

By assuming the expansion of a spherically symmetric flow centered at the limb, the maximum shift observable would be about $\pm 1.6 \AA$ for the O VI lines at exposure 2 . Although this CME expansion could actually account for the observed broad profiles, this interpretation is contradicted by the fact that the broadening does not increase in the next exposure. In fact, if the bulk velocity dominates, the lines should be much wider in exposure 3 than they are in exposure 2 because of the increase in the angle. The broad profiles in exposure 3 can be instead modeled as well by a similar model as for exposure 2 (see Table 2 ).

The interpretation of the observed broadening in terms of shocked plasma is also supported by the fact that the oxygen line profiles observed in exposure 1 (Fig. 6) already show broad wings (this feature is not present in

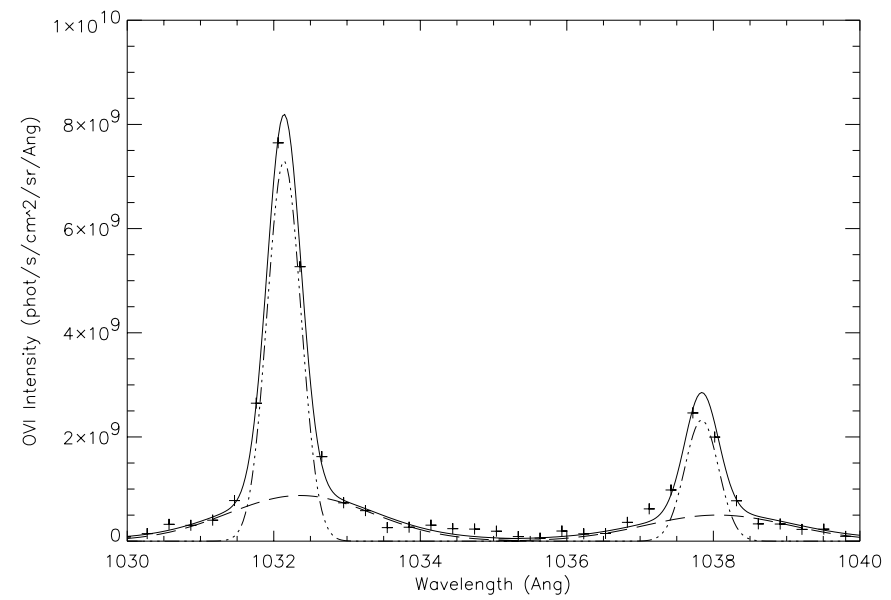

Fig. 6. UVCS March 3, 2000 observations of O VI $\lambda 1032$ and $\lambda 1037$ profiles at $1.7 R_{\odot}$ in the time interval 02:19:0702:22:07 UT (exposure 1). The intensity has been averaged over $9^{\prime}$ between $360^{\prime \prime}$ and $906^{\prime \prime}$ in the southern portion of the slit. The curves have the same meaning as in Fig. 5.

previous exposures at $1.6 R_{\odot}$ ) that can be modeled with the same mechanism used in Sect. 3.2 (see Table 2). Moreover, according to a simple linear extrapolation (Fig. 3), the CME front position at the last minute of exposure 1 was at about $1.55 R_{\odot}$. Even accounting for the error in estimating the CME leading edge position from the LASCO images (about $0.1 R \mathrm{~s}$ ), it is highly improbable that we could observe a contribution from the CME material in the spectra of exposure 1 that were taken at an average distance of $1.8 R_{\odot}$.

We then conclude that the line broadening observed in all three exposures represents a signature of the heating of the shocked plasma.

\section{Summary and conclusions}

The main goal of this paper was the study of the ion heating mechanism behind a coronal shock through the analysis of the observed spectral profiles of the shocked plasma. For this purpose, we investigated a $1100 \mathrm{~km} \mathrm{~s}^{-1}$ shock observed by UVCS on March 3, 2000. By comparing the relative positions of the $\mathrm{CME}$ leading edge as extracted from LASCO images and UVCS observations, we deduced that the shock was ahead of the CME leading edge. The shock speed was inferred by analyzing the drift rate of 
the emission bands in the radio dynamic spectra and using the density profile obtained from UVCS observations just before the passage of the shock. The shock was found to propagate about $20 \%$ faster than the projected CME speed, a result that is in agreement with theoretical models of CME-driven fast mode shocks.

The UVCS spectral lines were strongly Doppler dimmed and broadened a few minutes after the passage of the shock. The strong Doppler dimming was attributed to the abrupt acceleration of the ions and to the temperature increase of the shocked plasma. The presence of extended wings in the post-shock O VI doublet profiles was attributed to a mechanism of nonadiabatic heating of the ions not deflected by the shock front. As a consequence, the observed line broadening is related to the gyration velocity of the ions in the downstream region, acquired through the conversion of part of the upstream normal velocity across the thin shock ramp. These results are consistent with in situ measurements of larger heating of the less abundant ion species respect to the protons downstream of interplanetary shocks (Berdichevsky et al. 1997).

This shock can be compared to the June 11, 1998 event observed by Raymond et al. (2000). Both shocks were characterized spectroscopically by a sudden broadening of the O VI line profiles and a doubling of the Si XII line intensity, a result that is consistent with shock heating and plasma compression. Raymond et al. (2000) found however a significant difference between the shock speed inferred from SOHO observations and the speed derived from the type II drift rate. In the March 3, 2000 event the shock was detected by UVCS ahead of the CME leading edge propagating at a speed consistent with theoretical models of CME-driven shocks. However, even in this case a blast wave origin cannot be completely discarded due to the fact that the Wind spacecraft (Bougeret et al. 1995) did not detect an associated interplanetary signature of a type II radio burst.

In spite of the problems related with the determination of the shock origin, we have now shown that the observation of the ultraviolet line profiles from shocked coronal plasma can provide an important diagnostic for the mechanisms of heating behind a coronal shock.

Acknowledgements. The authors are grateful to the EIT, LASCO, Hiraiso, Culgoora and BIRS consortia for the provision of supporting images. SOHO is a project of international cooperation between ESA and NASA.

\section{References}

Berdichevsky, D., Geiss, J., Gloeckler, G., \& Mall, U. 1997, J. Geophys. Res., 102, 2623

Bougeret, J.-L., Kaiser, M. L., Kellogg, P. J., et al. 1995, Space Sci. Rev., 71, 231

Brueckner, G. E., Howard, R. A., Koomen, M. J., et al. 1995, Sol. Phys., 162, 357

Delaboudinière, J.-P., Artzner, G. E., Brunaud, J., et al. 1995, Sol. Phys., 162, 291

Dodero, M. A., Antonucci, E., Giordano, S., \& Martin, R. 1998, Sol. Phys., 183, 77

Erickson, W. C. 1997, Publ. Astron. Soc. Aust., 14, 278

Gabriel, A. H., Garton, W. R. S., Goldberg, L., et al. 1971, ApJ, 169, 595

Gardner, L. D., et al. 1996, Proc. SPIE, 2381, 2

Hyder, C. L., \& Lites, B. W. 1970, Sol. Phys., 14, 147

Kahler, S. W., Raymond, J. C., \& Laming, J. M. 1999, in Solar Wind Nine, AIP Conf. Proc., 471, 685

Kohl, J. L., \& Withbroe, G. L. 1982, ApJ, 256, 263

Kohl, J. L., Esser, R., Gardner, L. D., et al. 1995, Sol. Phys., 162,313

Kohl, J. L., Noci, G., Antonucci, E., et al. 1997, Sol. Phys., 175,613

Kohl, J. L., Esser, R., Granmer, S. R., et al. 1999, ApJ, 510, L59

Kondo, T., Isobe, T., Igi, S., Watari, S., \& Tokumaru, M. 1995, J. Commun. Res. Lab., 42, 111

Lee, L. C., Wu, C. S., \& Hu, X. W. 1986, Geophys. Res. Lett., 13, 209

Lee, L. C., Mandt, M. E., \& Wu, C. S. 1987, J. Geophys. Res., 92,13438

Lee, L. C., \& Wu, B. H. 2000, ApJ, 535, 1014

Li, X., Habbal, S. R., Kohl, J., \& Noci, G. 1998, ApJ, 501, 133

Maxwell, A., Dryer, M., \& McIntosh, P. 1985, Sol. Phys., 97, 401

McLean, D. J. 1967, Proc. Astron. Soc. Austral., 1, 47

Newkirk, G. A. 1961, ApJ, 133, 983

Nelson, G. J., \& Melrose, D. B. 1985, in Solar Radiophysics, ed. D. J., McLean, \& N. R., Labrum (Cambridge Univ. Press)

Noci, G., Kohl, J. L., \& Withbroe, G. L. 1987, ApJ, 315, 706

Prestage, N. P., Luckhurst, R. G., Peterson, B. R., Bevins, C. S., \& Yuile, C. G. 1994, Sol. Phys., 150, 393

Raymond, J. C., Thompson, B. J., St. Cyr, O. C., et al. 2000, Geophys. Res. Lett., 27, 1439

Smerd, S. F., Sheridan, K. V., \& Stewart, R. T. 1975, ApJ, 16, L23S

Vernazza, J. E., \& Reeves, E. M. 1978, ApJS, 37, 485

Wild, J. P. 1950, Aust. J. Sci. Res., A3, 541

Woods, T. N., Rottman, G. J., Bailey, S. M., Solomon, S. C., \& Worden, J. R. 1998, Sol. Phys., 177, 133 